\title{
A Study of Authenticated Communication Based on Magic Square and Goldbach's Conjecture
}

\author{
Hui-Shan Li, Chenglian Liu, Member, IEEE, and Sonia C-I Chen, Member, IEEE
}

\begin{abstract}
Although the magic square is a historical and universal study, its progress has been limited, to numeric games, which is closer to digital games or word games, and lacks the connection with mainstream mathematics. Recently, its study has extended from exciting mathematical games to various novel applications, such as image encryption, decryption processing, watermarking solutions, and student group learning problems, or different engineering applications. In terms of employment in information security, it is the blue ocean that requires more innovative research to enrich its content. In this study, we engage the magic square and Goldbach's Conjecture to develop an innovative method to search prime numbers.
\end{abstract}

Keywords-Magic Square, Mutation, Goldbach's Conjecture, Authenticated Communication, Cybersecurity

\section{INTRODUCTION}

The history of the magic square can be traced back to ancient China 2000 years ago [1]. Later, it has been stretched to Japan, India, the Middle East, and Latin Europe [2]-[6]. Despite its long history and widespread, its progress has been limited to mathematic games, which is closer to digital games or word games. It lacks the connection with mainstream mathematics and theoretic disciplines [7], [8]. Recently, its study has extended from exciting mathematical games to various novel and pragmatic subjects, such as image encryption [9], [10], decryption processing [11], [12], watermarking solutions [13], and student group learning issues [14], [15], or different engineering applications [16]-[18]. Owning to the features of the Magic Squares, it has been employed in an information hiding scheme. Huang [19] applied magic square to data hiding in 2007. Wen, Huang and Han used a sequential magic square, reordered the exchanged data by the transposition square. Both transposition and substitution are performed in the encryption and decryption processes in operation [20]. Sequentially, Shu [21] applied magic square to the pixel modification data hiding. Shen [22] and Liu et al. [23] implanted magic square to a scrambling matrix such as a block, and combined stream cipher to encrypt and decrypt the randomaccess files. The advance of data hiding, such as robust and secure data hiding techniques for telemedicine applications, has enhanced the security development in connected health care [24]-[29]. It is well known that an odd magic square is easier to create than an even square. The question then

Mr. Li is with Computer and Network Center, Fujian Polytechnic Normal University, Fuqing 350300, China. E-mail: (lhs1980@163.com).

Dr. Liu is with the School of Computing, Neusoft Institute of Guangdong, Foshan 528225, China. Corresponding author, E-mail: (chenglian.liu@gmail.com).

Dr. Chen is with School of Economics and Finance, Huaqiao University, Xiamen 361021, China. E-mail: (drsoniachen@mail.com)

Manuscript received January xx, 2020; revised xx.xx, 2020. becomes, can you use an odd magic square to produce an even number. Liu has built up fundamental knowledge according to the key points mentioned above to enrich the employment of magic squares in the domain of information security [30]. This study based on the fundamental knowledge of magic square [31]-[34], and further engages it with Goldbach's Conjecture to develop an innovative method of authenticated communication. The structure of this paper is organized into several sections. Section 1 introduces the brief history of the magic square and the current development. Section 2 describes the properties of the magic square, mutation to the magic square, and the relationship of Goldbach's conjecture with magic square. Section 3 describes the characteristics and extension of the application of the magic square, which used in encryption, decryption, and authentication for a network environment. Finally, the conclusion is drawn in section 4.

\section{Analysis of Magic Square Mutation}

\subsection{Related Works and Literature Review}

In this paper, the author lists some literatures which discuss the application of magic square in other fields, such as image processing, data and information hiding, watermarking, information security, and other application issues. Due to limited conditions, this study lists parts of good contributions, but is a little different then what is discussed in this article, please see Table 1.

Table 1

RELATED LITERATURES

\begin{tabular}{|c|c|c|c|}
\hline Year Image & Data Hiding & Watermarking & Other Application \\
\hline 2004 & & & Abiyev et al. [16] \\
\hline 2007 & Huang [19] & & \\
\hline 2009 & & & Chang et al. [35] \\
\hline 2010 & Wen [20] & & Ganapathy [34] \\
\hline 2011 Hsu [21] & Hsu [21] & & Shen [22], Liu et al. [23] \\
\hline 2012 Zhang [36] & & & Lee [37], Liu et al. [38] \\
\hline 2014 & & Channapragada [39] & Duan $[40]$ \\
\hline 2015 & & & Duan [41] \\
\hline 2015 & & & Liu [30], Liu et al. [42] \\
\hline \multicolumn{4}{|c|}{2016 Zhong et al. [43] Kurup et al. [24] } \\
\hline \multicolumn{4}{|c|}{2016 Rao [32], [33] } \\
\hline \multicolumn{4}{|c|}{2017 Sowmiya [31] } \\
\hline 2018 & & & Chia [44] \\
\hline 2019 & & & Woll $[45]$ \\
\hline 2020 & & & Peng et al. [46] \\
\hline
\end{tabular}

\subsection{Magic Square History}

According to literature, the oldest magic square is a 3-order magic square from ancient Chinese Lo-Shu. On the basis of legend, Dayu governance flooding in the Yellow River and Luo-Shui; he found a turtle's rear engraved peculiar pattern in 
a standard 3-order magic square [47]-[50]. The magic square is also known as "Jiugong calculation", a total of nine blocks because sum of row or column numbers are equivalent to this matrix. In Japan, the magic square is also called as "Kyu Miya San" [47], [48].

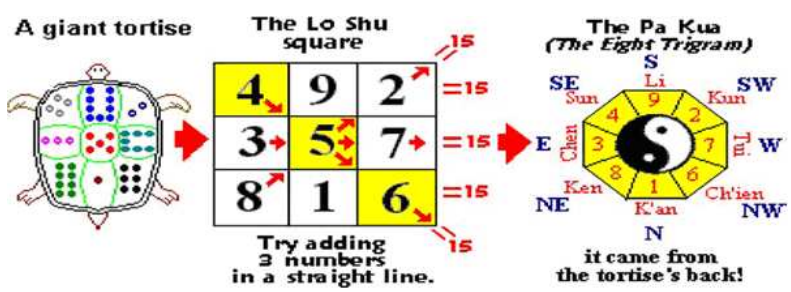

Figure 1. A 3-Order Magic Square in Turtl's Rear [16].

\section{Notations:}

$n$ : express an positive integer.

$p$ : express an integer.

$q$ : express an integer.

$p_{1}$ : express a prime.

mod: express the modulo operation.

\subsection{Definition of Magic Square}

If a matrix have $n$ rows and $n$ straights, and there have $n$ squared small squares, then this matrix is an n-order square; if each column, each row and each diagonal digital numbers are equal, its called a standard magic square (or normal magic square) [49], [50]. How to check a magic square, we could calculate the sum of each row, column or diagonal where the formula is

$$
\frac{n^{3}+n}{2},
$$

wherein $n$ is a order for this magic square.

For example: A case of 3-order magic square, the sum of each row, column or diagonal is $\frac{3^{3}+3}{2}=15$.

\begin{tabular}{|l|l|l|}
\hline 8 & 1 & 6 \\
\hline 3 & 5 & 7 \\
\hline 4 & 9 & 2 \\
\hline
\end{tabular}

Figure 2. A Standard 3-order Magic Square.

\subsection{Mutation of Magic Square}

There are several common deformations to magic square: the rigid deformation, the value-added deformation, the complementary deformation, the tic-tac-toe exchange deformation, the swastika deformation, the topological deformation, the rotation deformation and so on variety way [47]. To deform an odd or even magic square, those are different methods. In this paper, we would like introduce a case of synthesis to 9-order and 12-order magic square.
1) Odd Number of 9-Order Magic Square Synthesis Method: To a n-order magic square can be sliced several porders or q-orders where

$$
n=p \cdot q, n \geq 3
$$

Then this $n$ be factored two integers $p$ and $q$. For example, a 9 -order magic square can slit 9 parts 3 -order magic square as shown in Figure 3.

\begin{tabular}{|l|l|l|l|l|l|l|l|l|l|}
\hline 71 & 64 & 69 & 8 & 1 & 6 & 53 & 46 & 51 \\
\hline 66 & 68 & 70 & 3 & 5 & 7 & 48 & 50 & 52 \\
\hline 67 & 72 & 65 & 4 & 9 & 2 & 49 & 54 & 47 \\
\hline 26 & 19 & 24 & 44 & 37 & 42 & 62 & 55 & 60 \\
\hline 21 & 23 & 25 & 39 & 41 & 43 & 57 & 59 & 61 \\
\hline 22 & 27 & 20 & 40 & 45 & 38 & 58 & 63 & 56 \\
\hline 35 & 28 & 33 & 80 & 73 & 78 & 17 & 10 & 15 \\
\hline 30 & 3 & 34 & 75 & 77 & 79 & 12 & 14 & 16 \\
\hline 31 & 36 & 29 & 76 & 81 & 74 & 13 & 18 & 11 \\
\hline
\end{tabular}

Figure 3. 9-Order Magic Square Cutting-Synthesis

\section{The synthesis method of the type $3 * 3$}

Step 1. The matrix cuts three parts, it could be sliced nine medium blocks. The medium block also slice nine small blocks. We called tic-tac-toe cutting. This method looks like a Chinese word 'well'. that's why we named.

Step 2. For medium blocks, we marked 1-9 numbers to each blank according to 3-order style.

Step 3. There are 81 small blocks, we sequence fill out all numbers.

Step 4. Repeat step 3 until all small blocks is not empty.

Step 5. Check the sum of rows, columns and diagonals. If its not hold, repeat step 3, otherwise terminal these stages.

2) Even number of 12-order magic square Synthesis Method: A n-order magic square where $n=12$ can be divided two types $n=4 \cdot 3$ or $n=3 \cdot 4$, the matrix average slice three or four parts.

The synthesis method of type $4 * 3$

Step 1. A 12-order magic square average cut four equal parts, there are 16 medium blocks.

Step 2. Each medium blocks could be sliced 9 small blocks. So, there are totally 144 small blocks in this matrix.

Step 3. The medium blocks sequence marked 1-16 numbers, and small blocks sequence 1-9 numbers. We sequence fill out all numbers.

Step 4. Repeat step 3 until 144 small blocks is not empty.

Step 5. Check the sum of rows, columns and diagonals. If its not hold, repeat step 3, otherwise terminal these stages.

The result show in Figure 4.

The synthesis method of the type $3 * 4$

Step 1. A 12-order magic square average cut three equal parts, there are 9 medium blocks.

Step 2. Each medium blocks could be sliced 16 small blocks. So, there are totally 144 small blocks in this matrix. 


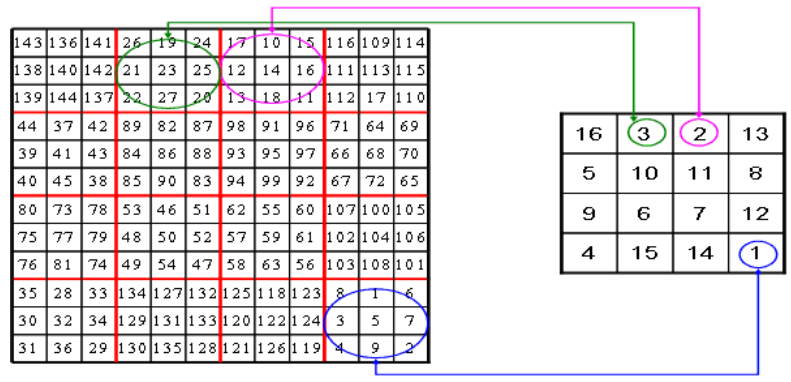

Figure 4. 12-Order Magic Square slicing in $4 * 3$

Step 3. The medium blocks sequence marked 1-9 numbers, and small blocks sequence 1-16 numbers. We sequence fill out all numbers.

Step 4. Repeat step 3 until 144 small blocks is not empty.

Step 5. Check the sum of rows, columns and diagonals. If its not hold, repeat step 3, otherwise terminal these stages.

The result show in Figure 5.

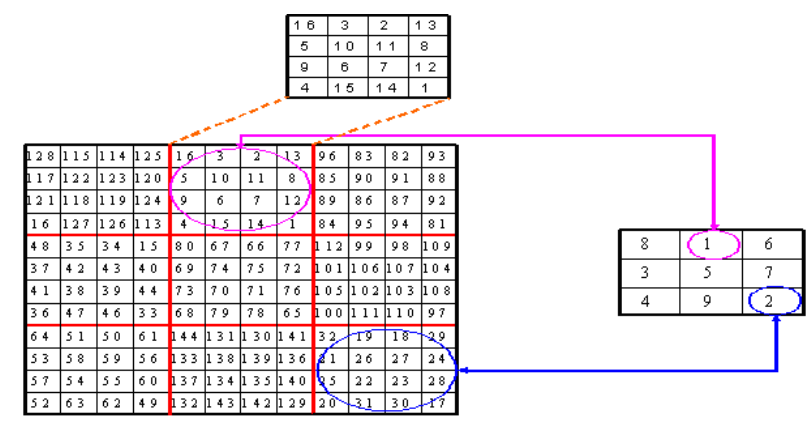

Figure 5. 12-Order Magic Square slicing in $3 * 4$

\section{The synthesis method of the type $3 * 5$}

Step 1. A 15-order magic square average cut three equal parts, there are 9 medium blocks.

Step 2. Each medium blocks could be sliced 25 small blocks. So, there are totally 225 small blocks in this matrix.

Step 3. The medium blocks sequence marked 1-9 numbers, and small blocks sequence 1-25 numbers. We sequence fill out all numbers.

Step 4. Repeat step 3 until 225 small blocks is not empty.

Step 5. Check the sum of rows, columns and diagonals. If its not hold, repeat step 3, otherwise terminal these stages.

The result show in Figure 6.

3) Note synthesis: As well known, the smallest known magic square is a 3 -order magic square, if we want to construct more than a 9-order magic square by synthesis and cutting method, the minimum requirement is a 3-order. Why not use 2-order? Because the 2-order magic square does not exist.

Proposition 1. Assumption a two-order magic square shows in Figure 7, The parameters $a, b, c, d$ are not equality positive integers.

Proof: We assume the Figure 7 is a 2-order magic square.

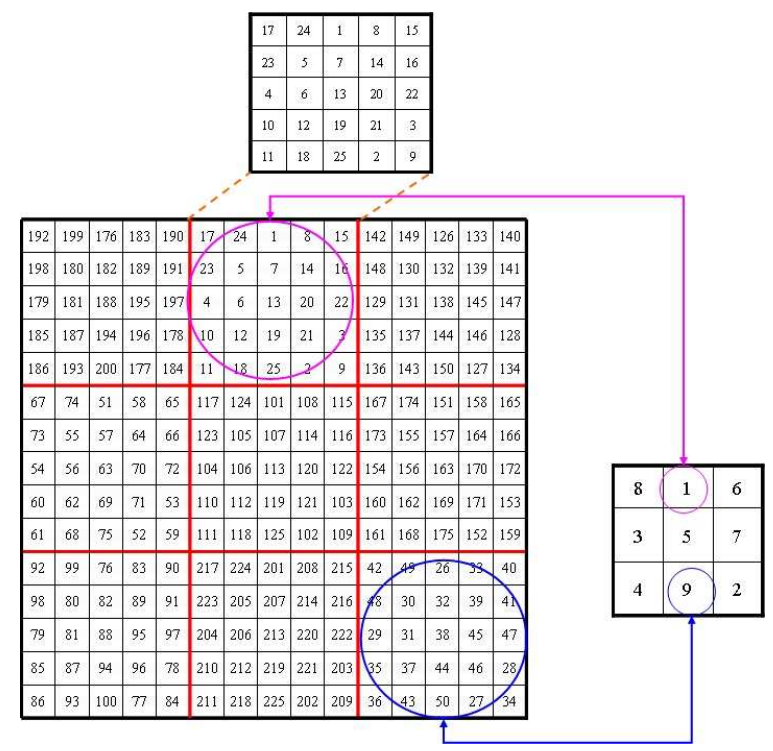

Figure 6. 15 -Order Magic Square slicing in $3 * 5$

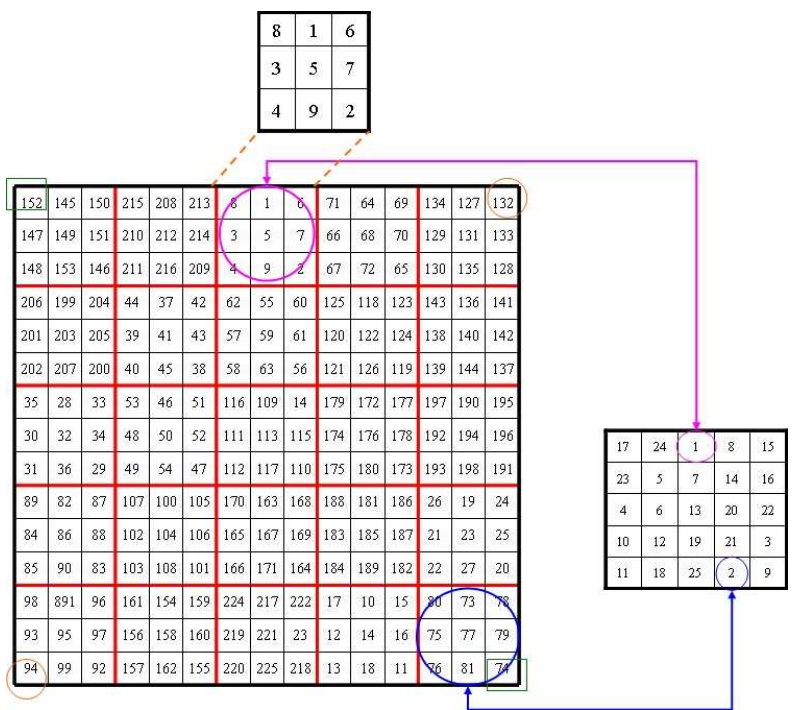

Figure 7. 15 -Order Magic Square slicing in $5 * 3$

We therefore know

$$
a+b=b+d=a+c=b+d=a+d=b+c
$$

, and obtain

$$
a=b=c=d .
$$

However, in definition of magic square, the necessary condition is

$$
a \neq b \neq b \neq d .
$$

Thus, it contradicts the assumption.

Proposition 2. Assume $n$ is a positive even (integer) number, if $n$ can be factored by 3 or 4 , it then could reconstruct from 3-order or 4-order magic square.

Proof: If $3 \mid n$ then $n \equiv 0(\bmod 3)$. If $4 \mid n$ then $n \equiv 0$ $(\bmod 4)$.

There are two situations in following: 


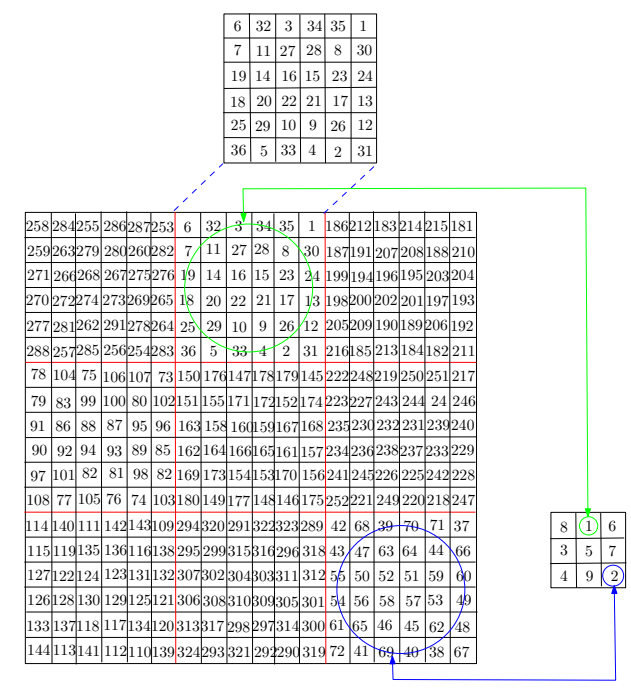

Figure 8. 18-Order Magic Square slicing in $3 * 6$

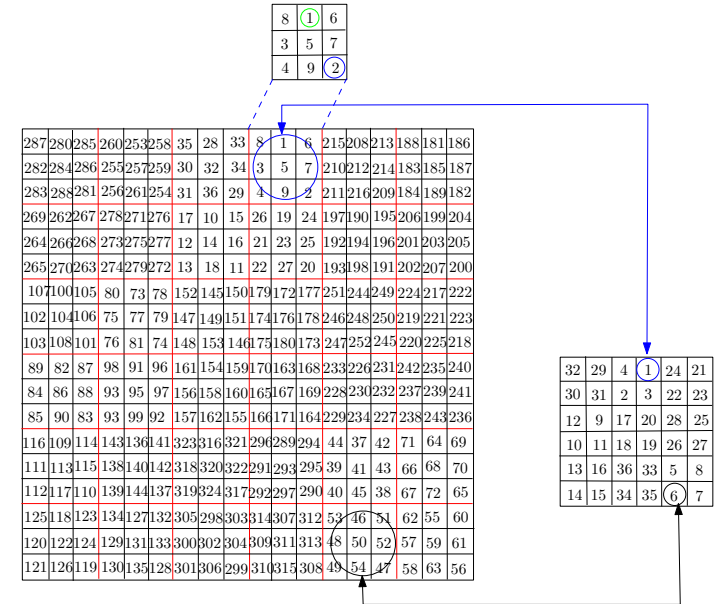

Figure 9. 18-Order Magic Square slicing in 6*3

\begin{tabular}{|l|l|}
\hline$a$ & $b$ \\
\hline$c$ & $d$ \\
\hline
\end{tabular}

Figure 10. A Two Dimensions Matrix

$$
(n) \Longleftrightarrow \begin{cases}\equiv 1 & (\bmod 3) \\ \equiv 2 & (\bmod 4),\end{cases}
$$

or

$$
(n) \Longleftrightarrow \begin{cases}\equiv 2 & (\bmod 3) \\ \equiv 2 & (\bmod 4) .\end{cases}
$$

We obtain this $n$ to match $2 p_{1} \mid n$ where $p_{1}$ is prime. Thus, the prime $p_{1}$ can not factoring by any integer. And $2 p_{1}$ is an even number, it does not exist any factors of 3 or 4 .

If $n$ is an integer, there are six types: $\{0-0\},\{2-2\}$, $\{1-0\},\{0-2\},\{2-0\}$ and $\{1-2\}$, we obtained this result from Table 2. If and only if $p_{1}$ is a prime where $\frac{n}{2}=p_{1}$ and $n \geq 7$, there are filtered three cases, namely $\{2-2\},\{0-2\}$ and $\{1-2\}$ forms. However the form of $\{0-2\}$ is divided
Table 2

\begin{tabular}{|c|c|c|c|}
\hline integer $n \quad \bmod$ & 3 & 4 & note \\
\hline 0 & 0 & 0 & \\
\hline 1 & 1 & 1 & \\
\hline 2 & 2 & 2 & \\
\hline 3 & 0 & 3 & \\
\hline 4 & 1 & 0 & \\
\hline 5 & 2 & 1 & \\
\hline 6 & 0 & 2 & \\
\hline 7 & 1 & 3 & \\
\hline 8 & 2 & 0 & \\
\hline 9 & 0 & 1 & \\
\hline 10 & 1 & 2 & $\sqrt{ }$ \\
\hline 11 & 2 & 3 & \\
\hline 12 & 0 & 0 & $\sqrt{ }$ \\
\hline 13 & 1 & 1 & \\
\hline 14 & 2 & 2 & $\sqrt{ }$ \\
\hline 15 & 0 & 3 & \\
\hline 16 & 1 & 0 & $\sqrt{ }$ \\
\hline 17 & 2 & 1 & \\
\hline 18 & 0 & 2 & $\sqrt{ }$ \\
\hline 19 & 1 & 3 & \\
\hline 20 & 2 & 0 & $\sqrt{ }$ \\
\hline 21 & 0 & 1 & \\
\hline 22 & 1 & 2 & $\sqrt{ }$ \\
\hline 23 & 2 & 3 & \\
\hline 24 & 0 & 0 & \\
\hline 25 & 1 & 1 & \\
\hline 26 & 2 & 2 & $\sqrt{ }$ \\
\hline 27 & 0 & 3 & \\
\hline 28 & 1 & 0 & \\
\hline
\end{tabular}

AN INTEGER CHANGING SITUATIONS

by 3 , then $n$ is not a prime. Otherwise, it is a contradiction. Thus, two forms $\{2-2\}$ or $\{1-2\}$ probably be a prime where $\frac{n}{2}=p$.

Table 3

REDUCE EXPRESSION

\begin{tabular}{cccc}
\hline$n$ & $n \bmod 3$ & $n \bmod 4$ & $\frac{n}{2}$ \\
\hline 12 & 0 & 0 & 6 \\
14 & 2 & 2 & 7 \\
16 & 1 & 0 & 8 \\
18 & 0 & 2 & 9 \\
20 & 2 & 0 & 10 \\
22 & 1 & 2 & 11 \\
\hline
\end{tabular}

$\frac{n}{2}\left\{\begin{array}{l}\text { Case 1: this is an even number. } \\ \text { Case 2: this is an odd number. } \\ \text { Case } 3: \text { this is a float number. }\end{array}\right.$

We rewrite the Equation (8) as follow: 


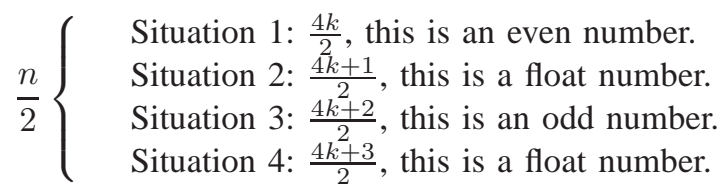

According to Bertrand, he assumes that if $n>3$, there is always at least one prime $p$ between $n$ and $2 n-2$ in 1845 . In other words, if $n>1$, then there is always at least one prime $p$ such that $n<p_{1}<2 n$. This postulation is also named the Bertrand-Chebyshev theorem or Chebyshev's theorem. As it was proven by Chebyshev using non-elementary methods in 1850 [51], [52].

Theorem 1 (Bertrand-Chebyshev Theorem). For any real number $n$, where $n \geq 1$, there always exists at least a prime between the interval $n$ and $2 n$.

Proof: We suppose that

$$
\begin{aligned}
\left(\begin{array}{c}
2 n \\
n
\end{array}\right) & \leq \prod_{p \leq \sqrt{2 n}} P^{r} \prod_{\sqrt{2 n<p \leq \frac{3}{2} n}} P \prod_{m<p \leq 2 n} P \\
& \leq \prod_{p \leq \sqrt{n}}(2 n) \prod_{\sqrt{2 n}<p \leq m} P \prod_{m<p<2 m} P .
\end{aligned}
$$

For each $n$, where $1 \leq n<4010$, such as $2,3,5,7,13,23$, 43, 83, 163, 317, 631, 1259, 2503, .., 3967, 3989, 4001, 4003, 4007.

We choose a small prime $p_{1}$, and another greater than $n$ say $p_{1}^{\prime}$. The relationship is as follows:

$$
p_{1} \leq n \leq p_{1}^{\prime} \leq 2 p_{1} \leq 2 n .
$$

Thus, this finishes the proof.

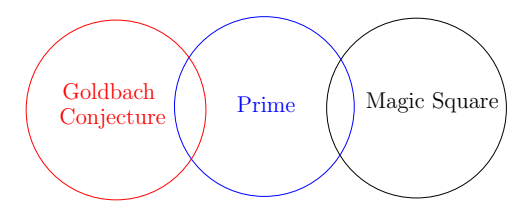

Figure 11. A relationship among Goldbach conjecture, prime, and the magic square

We get following relationship as:

Goldbach's conjecture $\cap$ prime $\cap$ magic square.

\section{ENCRYPTION AND DECRYPTION PRINCIPLE AND ITS AUTHENTICATION MECHANISM}

\subsection{Magic square characteristics-"greater than 1 rule"}
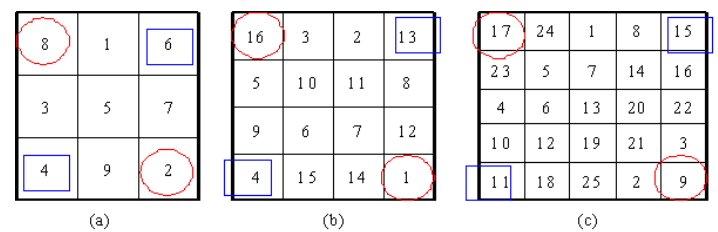

Figure 12. (a) 3-Order Magic Square. (b) 4-Order Magic Square. (c) 5-Order Magic Square.
3-Order magic square features: An example for $3 * 3$ magic square, the sum of two diagonal corners are $(8+2)=10$ and $(6+4=10)$, we know ten is greater 1 than nine.

4-Order magic square features: An example for $4 * 4$ magic square, the sum of two diagonal corners are $(16+1)=17$ and $(13+4)=17$, we know seventeen is greater 1 than sixteen.

5-Order magic square features: An example for $5 * 5$ magic square, the sum of two diagonal corners are $(17+9)=26$ and $(15+11)=26$, we know twenty-six is greater 1 than twenty-five.

\begin{tabular}{|c|c|c|c|c|c|}
\hline 6 & 22 & 3 & 34 & 35 & 1 \\
\hline 7 & 11 & 27 & 28 & 8 & 30 \\
\hline 19 & 14 & 16 & 15 & 23 & 24 \\
\hline 18 & 20 & 22 & 21 & 17 & 13 \\
\hline 25 & 29 & 10 & 9 & 26 & 12 \\
\hline \hline 36 & 5 & 33 & 4 & 2 & 31 \\
\hline \multicolumn{5}{|c}{ (a) } \\
\hline
\end{tabular}

\begin{tabular}{|c|c|c|c|c|c|c|}
\hline 30 & 39 & 48 & 1 & 10 & 19 & 28 \\
\hline 38 & 47 & 7 & 9 & 18 & 27 & 29 \\
\hline 46 & 6 & 8 & 17 & 26 & 35 & 37 \\
\hline 5 & 14 & 16 & 25 & 34 & 36 & 45 \\
\hline 13 & 15 & 24 & 33 & 42 & 44 & 4 \\
\hline 21 & 23 & 32 & 41 & 43 & 3 & 12 \\
\hline 22 & 31 & 40 & 49 & 2 & 11 & 20 \\
\hline \multicolumn{6}{|c|}{ (b) } \\
\hline
\end{tabular}

\begin{tabular}{|c|c|c|c|c|c|c|c|}
\hline 64 & 2 & 3 & 61 & 60 & 6 & 7 & 57 \\
\hline 9 & 55 & 54 & 12 & 13 & 51 & 50 & 16 \\
\hline 17 & 47 & 46 & 20 & 21 & 43 & 42 & 24 \\
\hline 40 & 26 & 27 & 37 & 36 & 30 & 31 & 33 \\
\hline 32 & 34 & 35 & 29 & 28 & 38 & 39 & 25 \\
\hline 41 & 23 & 22 & 44 & 45 & 19 & 18 & 48 \\
\hline 49 & 15 & 14 & 52 & 53 & 11 & 10 & 56 \\
\hline 8 & 58 & 59 & 5 & 4 & 62 & 63 & 1 \\
\hline \multicolumn{8}{|c}{ (c) } \\
\hline
\end{tabular}

Figure 13. (a) 6-Order Magic Square. (b) 7-Order Magic Square. (c) 8-Order Magic Square.

6-Order magic square features: For $6 * 6$ magic square, the sum of two diagonal corners number are $(6+31)=37$ and $(1+36)=37$, we know thirty-seven is greater 1 than thirty-six. 7-Order magic square features: An example for $7 * 7$ magic square, the sum of two diagonal corners are $(30+20)=50$ and $(28+22)=50$, we know fifty is greater 1 than forty-nine.

8-Order magic square features: An example for $8 * 8$ magic square, the sum of two diagonal corners are $(64+1)=65$ and $(57+8)=65$, we know sixty-five is greater 1 than sixty-four.

9-Order magic square features: An example for $9 * 9$ magic square, the sum of two diagonal corners are $(71+11)=82$ and $(51+31)=82$, we know sixty-five is greater 1 than eighty-two. 12-Order magic square features: An example for $12 * 12$ magic square, the sum of two diagonal corners are $(143+2)=145$ and $(114+31)=145$, we know 145 is greater 1 than 144.

12-Order magic square features: An example for $12 * 12$ magic square, the sum of two diagonal corners are $(128+17)=145$ and $(93+52)=145$, we know 145 is greater 1 than 144.

15-Order magic square features: An example for $15 * 15$ magic square, the sum of two diagonal corners are $(192+34)=226$ and $(140+86)=226$, we know 225 is greater 1 than 226.

15-Order magic square features: An example for $15 * 15$ magic square, the sum of two diagonal corners are $(152+74)=226$ and $(132+94)=226$, we know 225 is greater 1 than 226.

18-Order magic square features: An example for $18 * 18$ magic square, the sum of two diagonal corners are $(258+67)=325$ and $(181+144)=325$, we know 325 is greater 1 than 324 .

18-Order magic square features: An example for $18 * 18$ magic square, the sum of two diagonal corners are $(287+56)=343$ and $(121+186)=307$, then $(343+307)=650$, and $(650 / 2)=325$. we know 325 is greater 1 than 324 . 
3.2. Authentication mechanism with the characteristics of the magic square

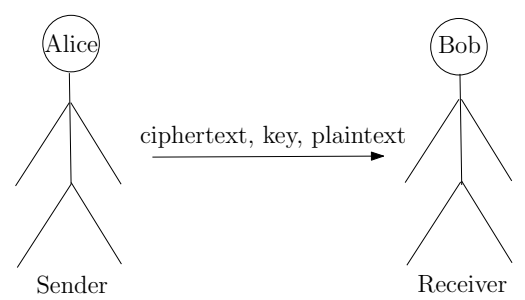

Figure 14. Sender-Receiver Single Channel Relationship.

In basic situation, if sender wants to communicate with receiver, he must send three parameters \{cipher, key, and plaintext $\}$ on an insecure signal channel. To receiver, when he received these parameters from sender. He can decrypt ciphertext to plaintext through by key. For symmetric cryptosystem mechanism. The encryption key is same with decryption key. To asymmetric cryptosystem, it is not the same key to encryption and decryption. How does receiver authenticate sender? Receiver decrypted ciphertext and then compared with original plaintext, if it is hold, he rather believes sender is a right person, otherwise the sender is an adversary. (see Figure 14)

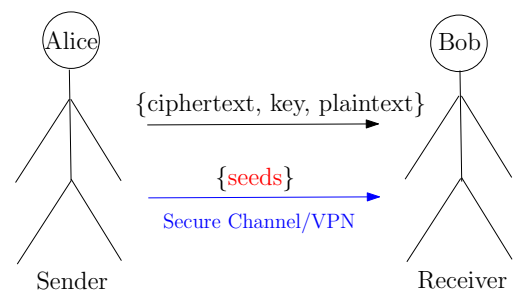

Figure 15. Sender-Received Two Channel Relationship.

If sender and receiver preshare a magic square algorithm. Sender send the ciphertext by common channel and seeds by secure channel (or Virtual Private Network, VPN) to receiver. In the mean time, sender does not send the plaintext to receiver. Receiver then can decrypt the plaintext by himself.

For example: The sender transmitted seeds $\{7,30,28,22,20\}$ via a secure channel seed and ciphertext to the receiver. When receiver catch these parameters, he knows to generate a 7-order magic square, and then compare four corners $\{30,28,22,20\}$. If it is hold, this magic square is correct, otherwise it is wrong. Receiver can not recover ciphertext from a wrong magic square. (see Figure 15)

\subsection{Security Analysis}

The 3-order magic square is unique, the 4-order has 880 variants, the 5-order owns 275305224 varieties, the 6-order calculated about $1.775399 \cdot 10^{19}$, and 10 -order estimated $2.4149 \cdot 10^{110}$ combinations. Such proof of permutations and combinations, be introduced in [50].

\section{Open Problems:}

1) Does "greater 1 rule" suit to most parts of magic square, how many magic square did not match for this rule? There is not any reference to discuss it.

2) There are so many articles to discuss how to generate a odd magic square, but fewer discuss how fast generate an even magic square.

3) Does magic square have widely application? Apply where? It is still a pending study.

\section{CONCLUSION}

According to the literature review, the magic square has shifted from mathematic games to various applications and theoretical mathematics analysis. This study further discusses its usage in cybersecurity authentication and the similarity with Goldbach conjecture to explore its value both in practice and theory. The employment of the magic square to encryption and decryption provides various possibilities for the block cipher. The sender still transmits the plaintext, key, and ciphertext to the receiver who can authenticate each other. In the method proposed in this study, the sender just needs to support ciphertext and seeds only, no need to transmit plaintext. The receiver can easily generate a magic square by himself and then use this matrix to encrypt/decrypt source messages. There are two strengths in this method: 1) Reduce the transmitting performance. 2) Against the known-plaintext attack where there is no plaintext and key in progress. To sum up, this study contributes to the knowledge of authenticated communication by proposing innovative methods based on magic square and Goldbach's conjecture. A variety of research, such as cybersecurity, and cryptography, can be developed based on the foundation of this study.

\section{ACKNOWLEDGMENTS}

The authors would like to thank the anonymous reviewers for their useful comments. This work was supported by Huaqiao University High-Level Talent Research Startup Project and The Straits Postdoctoral Fellowship Funding Program in 2018, Fujing Financial office no. [2017] 32/50X18137. This work is partially supported from Neusoft Institute of Guangdong project under the number NUIT2018-001, and also partially supported by student innovation training program under the grant number PDJH2019B0569. This work is partially supported from university project, the grant number is XH17019 and the project name is big data decision support platform based on public opinion of campus network by Fujian Polytechnic Normal University.

\section{REFERENCES}

[1] S. Cammann, "The magic square of three in old chinese philosophy and religion," History of Religions, vol. 1, no. 1, pp. 37-80, 1961.

[2] G. Abe, "Unsolved problems on magic squares," Discrete Mathematics, vol. 127 , no. 1, pp. 3-13, 1994.

[3] D. L. Anderson, "Magic squares: Discovering their history and their magic," Mathematics Teaching in the Middle School, vol. 6, no. 8, pp. 466-471, 2001.

[4] K. S. Sreeranjini and V. M. Mallayya, "Semi magic squares as a field," International Journal of Algebra, vol. 6, no. 26, pp. 1249-1256, 2012.

[5] V. Karpenko, "Two thousand years of numerical magic squares," Endeavour, vol. 18, no. 4, pp. 147-152, 1994. 
[6] R. Comes, The Transmisssion of Azarquiel's Magic Squares in Latin Europe, 01 2016, pp. 159-198.

[7] Y. Ku and N.-X. Chen, "Some theorems on construction of magic squares," Journal of the Franklin Institute, vol. 322, no. 5, pp. 253266, 1986.

[8] A. T. Benjamin and K. Yasuda, "Magic "squares" indeed!" American Mathematical Monthly, vol. 106, no. 2, pp. 152-156, February 1999.

[9] H. Zhang, , X.-F. Wang, L. Z.-H. Li, D.-H. Liu, and Y.-C. Lin, "A new image encryption algorithm based on chaos system," in IEEE International Conference on Robotics, Intelligent Systems and Signal Processing, vol. 2, 2003, pp. 778-782.

[10] Y.-P. Zhang, P. Xu, and Z.-X. Lin, "Research of image encryption algorithm based on chaotic magic square," in Advances in Electronic Commerce, Web Application and Communication, D. Jin and S. Lin, Eds., vol. 2. Springer, 2012, pp. 103-109.

[11] G. Ganapathy and K. Mani, "Add-on security model for public-key cryptosystem based on magic square implementation," Lecture Notes in Engineering and Computer Science, vol. 2178, pp. 317-321, October 2009.

[12] A. Dharini, R. Devi, and I. Chandrasekar, "Data security for cloud computing using RSA with magic square algorithm," International journal of innovation and scientific research, vol. 11, pp. 439-444, 2014.

[13] H. Wang and Y.-L. Ji, "The research of digital watermarking in DRM system," Computer and Information Technology, vol. 14, no. 3, pp. 6163+67, 2006, chinese edition.

[14] M. Sitompul, "The effect of magic-square game in improving 10th grader students' speaking achievement," State University Of Medan, Indonesia., 2015, Undergraduate Thesis.

[15] D. D. Garcia and D. Ginat, "Demystifying computing with magic, continued," in Proceeding of the 44th ACM Technical Symposium on Computer Science Education, 2013, pp. 207-208.

[16] A.-A. Abiyev, A. Baykasoglu, T. Dereli, I. H. Filiz, and A. Abiyev, "Investigation of center of mass by using magic squares and its possible engineering applications," Robotics and Autonomous Systems, vol. 49, pp. 219-226, 2004

[17] D.-M. WANG, "The quasi-period of odd order magic square transformat ion on digital image," JournaL of Zhejiang University of Technology, vol. 33, no. 3, pp. 292-294, June 2005, chinese edition.

[18] Y. LIU and J. bo Liu, "Study and application of magic square group in process of image scrambling," Computer Technology and Development, vol. 22, no. 9, pp. 119-122, 2012, chinese Edition.

[19] S.-C. Huang, "Magic matrix encryption for secure data hiding," Master's thesis, National Central University, Taiwan., June 2007.

[20] M.-G. Wen, S.-C. Huang, and C.-C. Han, "An information hiding scheme using magic squares," in 2010 International Conference on Broadband, Wireless Computing, Communication and Applications, 2010, pp. 556560.

[21] Y.-M. Hsu, "Magic square algorithm for optimization of pixel pair modification data hiding," Master's thesis, National Taichung University of Science and Technology, Taiwan, June 2011.

[22] Y.-J. Shen, "A study of magic square based on transmission and encryption," Fuqing Brach of Fujian Normal University, China., 2011, Undergraduate Thesis.

[23] C. Liu, J.-M. Zhao, M. Rafsanjani, and Y. Shen, "A study on the stream cipher embedded magic square of random access files," in International Conference on Numerical Analysis and Applied Mathematics (ICNAAM), T. E. Simos, Ed., vol. 1389, 19-25 September 2011, pp. 1070-1073.

[24] S. Kurup, A. Rodrigues, and A. Bhise, "A data hiding technique based on magic squares," in 2016 International Conference on Data Mining and Advanced Computing (SAPIENCE), March 2016, pp. 175-179.

[25] A. K. Singh, B. Kumar, S. K. Singh, M. Dave, V. K. Singh, P. Kumar, S. P. Ghrera, P. K. Gupta, and A. Mohan, "Guest editorial: Robust and secure data hiding techniques for telemedicine applications," Multimedia Tools and Applications, vol. 76, no. 5, pp. 7563-7573, 2017.

[26] S. C.-I. Chen and C. Liu, "Factors influencing the application of connected health in remote areas, taiwan: A qualitative pilot study," International Journal of Environmental Research and Public Health, vol. 17, no. 4, p. 1282,2020

[27] Sonia Chien-I Chen, Chenglian Liu, and R. Hu, "Fad or trend? rethinking the sustainability of connected health," Sustainability, vol. 12, no. 5, p. 1775,2020

[28] S. C.-I. Chen, C. Liu, R. Hu, Y. Mo, and X. Ye, “"Nomen Omen": Exploring connected healthcare through the perspective of name omen," Healthcare, vol. 8, no. 1, p. 66, 2020.

[29] S. C.-I. Chen, R. Hu, and R. McAdam, "Smart, remote, and targeted health care facilitation through connected health: Qualitative study,"
Journal of Medical Internet Research, vol. 22, no. 4, p. e14201, April 2020.

[30] C. Liu, "A study of mutation magic square in authentication communication," in In Proceedings of the 9th International Conference on Computer Engineering and Applications, 2015, pp. 225-230.

[31] S. Sowmiya, I. M. Tresa, and A. P. Chakkaravarthy, "Pixel based image encryption using magic square," in 2017 International Conference on Algorithms, Methodology, Models and Applications in Emerging Technologies (ICAMMAET), Feb 2017, pp. 1-4.

[32] C. R. S. Rao and M. V. N. K. Prasad, Color Image Watermarking Techniques Based on Magic Square and Curvelets. Cham: Springer International Publishing, 2016, pp. 27-57.

[33] _ Color Image Watermarking Techniques Based on Magic Square and Ridgelets. Cham: Springer International Publishing, 2016, pp. 5974.

[34] G. Ganapathy and K. Mani, Information Security Enhancement to Public-Key Cryptosystem Through Magic Squares. Dordrecht: Springer Netherlands, 2010, pp. 423-437.

[35] C.-C. Chang, T. D. Kieu, Z.-H. Wang, and M.-C. Li, "An image authentication scheme using magic square," in 2009 2nd IEEE International Conference on Computer Science and Information Technology, 2009, pp. $1-4$.

[36] Y.-P. Zhang, P. Xu, and L.-Z. Xiang, Research of Image Encryption Algorithm Based on Chaotic Magic Square. Springer Berlin Heidelberg, 2012, pp. 103-109.

[37] M. Z. Lee, E. Love, S. K. Narayan, E. Wascher, and J. D. Webster, "On nonsingular regular magic squares of odd order," Linear Algebra and its Applications, vol. 437, no. 6, pp. 1346-1355, 2012.

[38] C. Liu, J. Lin, and S. Sun, "A study of encryption-decryption based on mutation magic square," Journal of Fuqing Branch of Fujian Normal University, vol. 113, no. 5, pp. 1-6, October 2012.

[39] R. S. R. Channapragada and M. V. N. K. Prasad, "Digital watermarking based on magic square and ridgelet transform techniques," in Intelligent Computing, Networking, and Informatics, D. P. Mohapatra and S. Patnaik, Eds., 2014, pp. 143-161.

[40] Z. Duan, J. Liu, J. Li, and C. Tian, "Improved even order magic square construction algorithms and their applications," in 8th International Conference on Combinatorial Optimization and Applications (CCOA), Z. Z. et al., Ed., 19-21 December 2014, pp. 668-680.

[41] _ - "Improved even order magic square construction algorithms and their applications in multi-user shared electronic accounts," Theoretical Computer Science, vol. 607, pp. 391-410, 2015.

[42] C. Liu, C.-C. Chang, Z.-P. Wu, and S.-L. Ye, "A study of relationship between RSA public key cryptosystem and Goldbach's conjecture properties," International Journal of Network Security, vol. 17, no. 4, pp. 445-453, July 2015.

[43] W. Zhong, Y. H. Deng, and K.-T. Fang, "Image encryption by using magic squares," in 2016 9th International Congress on Image and Signal Processing, BioMedical Engineering and Informatics (CISPBMEI), 2016, pp. 771-775.

[44] G. L. Chia, "Self-complementary magic squares of doubly even orders," Discrete Mathematics, vol. 341, no. 5, pp. 1359-1362, 2018.

[45] C. Woll, "There is a $3 * 3$ magic square of squares on the moon-A lot of them, Actually," The Mathematical Intelligencer, vol. 41, pp. 73-76, March 2019.

[46] C.-C. Peng, C.-J. Tsai, T.-Y. Chang, J.-Y. Yeh, and M.-C. Lee, "Novel heterogeneous grouping method based on magic square," Information Sciences, vol. 517, pp. 340-360, 2020.

[47] G.-X. LI, Magic Square Game. Xin Chao She Publishing Corporation, May 2009, vol. 1, Taipei, Taiwan.

[48] X.-B. Tan, An Interesting Magic Square. Fung Court Publishing Corporation, February 2004, vol. 1, Taipei, Taiwan.

[49] Wolfram Research Corporation, "Magic square," http://mathworld. wolfram.com/MagicSquare.html, April 2020.

[50] W. Trump, "How many magic squares are there?" http://www.trump.de/ magic-squares/howmany.html, 11 March 2019.

[51] Wolfram Research Corporation, "Bertrand's postulate," https://mathworld.wolfram.com/BertrandsPostulate.html, April 2020.

[52] Wikipedia, "Bertrand's postulate," https://en.wikipedia.org/wiki/ Bertrand_27s_postulate.

\section{Creative Commons Attribution License 4.0 (Attribution 4.0 International, CC BY 4.0)}

This article is published under the terms of the Creative Commons Attribution License 4.0

https://creativecommons.org/licenses/by/4.0/deed.en_US 